Pesq. Vet. Bras. 36(9):793-797, setembro 2016 DOI: 10.1590/S0100-736X2016000900001

\title{
Perfil da transmissão e prevalência da cisticercose bovina em propriedades rurais do Triângulo Mineiro ${ }^{1}$
}

\author{
Camilla T. Ducas Duarte ${ }^{2 *}$, Paulo Sérgio A. Pinto² ${ }^{2}$ Letícia F. Silva², Tatiane O. \\ Santos ${ }^{2}$, Emílio C. Acevedo-Nieto ${ }^{2}$ e Laerte P. Almeida ${ }^{3}$
}

\begin{abstract}
Duarte C.T.D., Pinto P.S.A., Silva L.F., Santos T.O., Acevedo-Nieto E.C. \& Almeida L.P. 2016. [Transmission and prevalence profile of bovine cysticercosis in rural properties of Triangulo Mineiro, Brazil.] Perfil da transmissão e prevalência da cisticercose bovina em propriedades rurais do Triângulo Mineiro. Pesquisa Veterinária Brasileira 36(9):793-797. Laboratório de Inspeção de Produtos de Origem Animal, Departamento de Medicina Veterinária, Universidade Federal de Viçosa, Avenida PH Rolfs 500, Viçosa, MG36570-000, Brazil.E-mail: camilla.ducas@ufv.br

Cysticercosis can affect cattle which develop the larvae Taenia saginata. Despite the importance of cysticercosis for public animal health and for the economy, the epidemiological reality of such zoonosis in Brazil is little known. In the southeastern region of the State of Minas Gerais most of the data are obtained from the official Sanitary InspectionServices in slaughterhouses, but little information based on field data from live animals. In order to assess the epidemiological situation of bovine cysticercosis in small and medium-sized rural properties of four municipalities, located in the region of Triângulo Mineiro, we searched the occurrence and the major risk factors associated with transmission of the disease in those properties. We conducted a cross-sectional epidemiological study involving 68 properties located in rural areas of the four municipalities, where there were collected 1002 bovine blood samples and applied an epidemiological questionnaire. The serological diagnosis of cysticercosis was conducted by sorting through indirect ELISA test, and the suspected cases were subjected to Immunoblot for confirmation. This study revealed the prevalence of $4.7 \%$ of bovine cysticercosis in rural properties of the municipalities sampled from July to August 2013. The factors which were statistically significant for the transmission of cysticercosis were the expertise of those responsible for the property (OR 5.81 IC 1,87-8.66), the family income (OR 6.02 IC 1.33-8.77) and the water quality of the property (OR 9.40 IC 1.97-9.59). Other factors observed were of lower significance. These results identified the main factors which interfere in the transmission of bovine cysticercosis, allowing to subsidize preventive measures and to control this important zoonotic disease in the region of Triângulo Mineiro.
\end{abstract}

INDEX TERMS: Taenia saginata, cysticercosis, serological, ELISA, Immunoblot, prevalence, risk factors, prevention, cattle.

RESUMO.- A cisticercose pode acometer o bovino, que desenvolve a larva da Taenia saginata. Apesar da importância da cisticercose para a saúde pública, para a saúde animal

\footnotetext{
${ }^{1}$ Recebido em 7 de julho de 2015.

Aceito para publicação em 26 de abril de 2016.

${ }^{2}$ Laboratório de Inspeção de Produtos de Origem Animal, Departamento de Medicina Veterinária, Universidade Federal de Viçosa (UFV), Avenida PH Rolfs 500, Viçosa, MG 36570-000, Brasil. *Autor para correspondência: camilla.ducas@ufv.br

${ }^{3}$ Departamento de Medicina Veterinária, Universidade Federal de Uberlândia (UFU), Avenida Pará 1720, Uberlândia, MG 38405-320, Brasil.
}

e para economia, a realidade epidemiológica da ocorrência dessa zoonose no Brasil é pouco conhecida. No estado de Minas Gerais a maioria dos dados é obtida dos serviços oficiais de Inspeção Sanitária nos matadouros-frigoríficos, sendo poucas as informações baseadas em dados de campo oriundos de animais vivos. Com o objetivo de avaliar a situação epidemiológica da cisticercose bovina em pequenas e médias propriedades rurais de quatro municípios, situados na região do Triângulo Mineiro, se pesquisou a ocorrência e os principais fatores de risco associados à transmissão da doença nessas propriedades. Dessa forma, foi realizado um 
estudo epidemiológico de corte transversal envolvendo as 68 propriedades localizadas nas zonas rurais dos quatro municípios, onde foram coletadas 1002 amostras de sangue bovino e aplicado um questionário epidemiológico. 0 diagnóstico sorológico da cisticercose foi realizado por triagem pelo teste ELISA indireto e os casos suspeitos foram submetidos ao Imunoblot para confirmação. Este estudo revelou a prevalência de $4,7 \%$ de cisticercose bovina nas propriedades da zona rural dos municípios amostrados, no período de julho a agosto de 2013. Os fatores que se mostraram estatisticamente significantes para a transmissão da cisticercose foram a expertiness do responsável pela propriedade (RC 5,81 IC 1,87-8,66), a renda familiar (RC 6,02 IC 1,33-8,77) e a qualidade da água da propriedade (RC 9,40 IC 1,97-9,59). Outros fatores foram observados com menor significância. Esses resultados identificaram os principais fatores que interferem na transmissão da cisticercose bovina, permitindo subsidiar medidas de prevenção e controle dessa importante zoonose na região do Triângulo Mineiro.

TERMOS DE INDEXAÇÃO: Taenia saginata, cisticercose, sorologia, ELISA, Imunoblot, prevalência, fatores de risco, prevenção.

\section{INTRODUÇÃO}

A cisticercose bovina é uma doença causada pela forma larvar da Taenia saginata que pode causar prejuízos econômicos, principalmente em áreas de produção animal, uma vez que as carcaças infectadas são condenadas no abate com base na inspeção sanitária (Silva 2005), além de limitar a possibilidade de exportação de carnes (Santos \& Barros 2009). Infecções provocadas pela forma adulta do mesmo cestoide (teníase) ocorrem no ser humano sempre que a carne bovina contaminada é consumida na forma mal aquecida de acordo com o Centro de Controle e Prevenção de Doenças (CDC 2013).

0 referido cestoide tem distribuição mundial, sendo a infecção importante em países como África, América Latina e alguns países do Mediterrâneo, mas as informações epidemiológicas sobre a doença ainda são incompletas (Rey 2008, Ogunremi \& Benjamin 2010). Entretanto, estudos têm sido realizados com o objetivo de traçar o perfil epidemiológico dessa zoonose tanto no Brasil como em outros países, incluindo avaliações de risco. É importante identificar e gerenciar o risco da infecção em animais, visando reduzir os prejuízos tanto no segmento produtor, como no consumidor, afim de prevenir perdas comerciais e garantir uma carne de qualidade, sem riscos à saúde humana (Calvo-Artavia et al. 2013a). O diagnóstico deve envolver o animal e o ambiente no qual ele está inserido, permitindo dessa forma direcionar medidas para prevenir a transmissão da doença em uma determinada área a partir desses fatores de riscos os quais podem variar de uma região para outra.

Os procedimentos oficiais de abate possibilitam o rastreamento e a identificação de áreas de alto risco onde determinada enfermidade possa ocorrer (Oliveira et al. 2013), no entanto, o diagnóstico realizado na inspeção post mortem em estabelecimentos de abate está sujeito a falhas, pois apresentam baixa sensibilidade, a qual varia em torno de 10 a $50 \%$ em animais com infecções discretas (Scandrett et al. 2009, Eichenberger et al. 2011, Calvo-Artavia et al. 2013b). 0 desenvolvimento de testes de imunodiagnóstico, como teste ELISA e Imunoblot, são recomendados como uma alternativa para o diagnóstico da inspeção post mortem, permitindo localizar focos da doença e direcionar as medidas de controle, especialmente a orientação dos procedimentos rotineiros de inspeção sanitária, por meio da identificação prévia de animais que apresentam a doença (Deckers \& Dorny 2010, Acevedo-Nieto et al. 2012).

A presença da cisticercose bovina na região do Triângulo Mineiro está sendo sistematicamente divulgada, porém o diagnóstico é realizado exclusivamente pela inspeção post mortem, sem agregar informações sobre a sua forma de transmissão e outros indicadores epidemiológicos (Almeida et al. 2002, Almeida et al. 2006).

Utilizando o imunodiagnóstico, a presente pesquisa teve por objetivo avaliar o perfil epidemiológico da cisticercose bovina e os principais fatores de risco associados à transmissão da forma parasitária de T. saginata na zona rural de quatro municípios da região do Triângulo Mineiro, Minas Gerais, Brasil.

\section{MATERIAL E MÉTODOS}

O estudo foi conduzido nos municípios de Iraí de Minas, Romaria, Grupiara e Douradoquara, situados na microrregião de Patrocínio, mesorregião do Triângulo Mineiro, sudeste do Brasil. A população total é estimada em 16.454 habitantes, representando um PIB médio de \$29.217,94 e um Índice de desenvolvimento humano médio de 0,71 (IBGE 2014a).

Para a escolha de cada município, padronizaram-se as populações menores que 10.000 habitantes e abaixo de 20.000 bovinos, apresentando a agropecuária como principal atividade econômica (IBGE 2014b). Os quatro municípios são constituídos por 671 propriedades rurais, com uma população bovina de 46.922 cabeças e predomínio da criação animal extensiva e semiextensiva, que é uma das características relevantes na transmissão da cisticercose bovina.

As propriedades foram visitadas com o propósito de coletar dados sanitários de campo (questionário) e amostras de sangue de bovinos, visando traçar o perfil epidemiológico da cisticercose bovina na região.

Para o cálculo do número de propriedades a serem pesquisadas, foi considerada a prevalência da doença estimada em 5\% e um nível de significância de 5\% (Epi Info, versão 3.5.1, WHO, 2008). As propriedades foram selecionadas por processo de amostragem aleatória simples por meio de sorteio e distribuídas por municípios, proporcionalmente ao total de propriedades. Assim, foi realizado um estudo epidemiológico de corte transversal (Almeida 2012) envolvendo 68 propriedades localizadas nas zonas rurais dos quatro municípios.

No período de julho a agosto de 2013, foram coletadas amostras de sangue de 1002 bovinos, por punção da veia jugular, dos animais com idade igual ou superior a três meses, excluindo as fêmeas gestantes. Posteriormente as amostras foram centrifugadas a $905 \mathrm{~g}$ e os respectivos soros foram estocados individualmente em micro tubos a $-20^{\circ} \mathrm{C}$.

Nas mesmas visitas às propriedades foi aplicado um questionário epidemiológico, previamente testado, por um mesmo entrevistador aos respectivos responsáveis, que assinaram um termo de consentimento. Os referidos questionários continham informações sobre as condições sanitárias das propriedades, re- 
lacionadas ao sistema de criação animal, higiene pessoal, padrão de alimentação e das instalações das pessoas, com a finalidade de determinar os fatores de risco associados a doença.

0 diagnóstico sorológico dos bovinos foi realizado por triagem, em triplicata, pelo ELISA indireto e os casos suspeitos (resultados positivos ao ELISA) foram submetidos ao Imunoblot para confirmação, seguindo as metodologias (Pinto et al. 2000, Pinto et al. 2001, Monteiro et al. 2006) utilizadas no Laboratório de Inspeção de Produtos de Origem Animal, do Departamento de Veterinária, na Universidade Federal de Viçosa, Viçosa-MG, Brasil.

Os dados epidemiológicos foram confrontados com os da prevalência da cisticercose bovina nas diferentes propriedades e municípios para a identificação dos fatores de risco associados à transmissão da cisticercose bovina nas propriedades amostradas.

As análises estatísticas foram realizadas no programa SPSS 17.0, procedendo a análise de regressão logística binária simples para cada variável, considerando $\mathrm{p}<0.20$. As variáveis significantes foram submetidas à regressão logística binária multivariada, pelo método Backward: LR, considerando $\mathrm{p}<0.05$, como base para o cálculo da Razão de Chances (RC), com um com intervalo de confiança de 95\% entre todas as variáveis que apresentaram diferença estatisticamente significante.

O protocolo desta pesquisa foi aprovado pelo Comitê de Ética em Pesquisa com Animais (Processo 26/2013) e pelo Comitê de Ética em Pesquisa em Humano (Processo de 300.140 / 2013) da Universidade Federal de Viçosa.

\section{RESULTADOS E DISCUSSÃO}

Nas 68 propriedades amostradas, 151 bovinos se apresentaram como suspeitos para cisticercose no teste ELISA indireto, confirmando-se pelo Imunoblot, 47 animais positivos. Assim os animais que foram amostrados, seguido dos respectivos resultados laboratoriais obtidos por município, detalhados no Quadro 1, indicaram uma prevalência de 4,7\% para a cisticercose bovina na zona rural dos quatro municípios situados na microrregião de Patrocínio-MG, no período de julho a agosto de 2013. Dentre o total de propriedades, observou-se uma positividade de 41,2\% dessas (28). Em relação às propriedades diagnosticadas com cisticercose bovina por município amostrado, Romaria foi o que apresentou maior positividade, 53,8\%, seguido por Grupiara, 45,4\%; Iraí de Minas, 37,0\% e Douradoquara, $35,3 \%$.

As características do sistema produtivo nas propriedades amostradas mostraram inicialmente que 45,6\% dessas eram pequenas e 54,4\% eram médias propriedades. Considerando o tipo de exploração produtiva, em 58,5\% das propriedades se produzia leite e carne. Com relação ao tipo de criação animal, na maior parte delas (85,3\%) praticava a cria, a recria e a engorda, sob criação extensiva. Os bovinos eram destinados ao abate não inspecionado em 36,8\% das propriedades. A alimentação dos animais era adotada à base de pastagem, e durante o período seco esses animais recebiam algum tipo de suplementação. A água consumida pelos animais nas propriedades era proveniente de mina/ nascente em 36,8\% dessas, em 33,8\% de rio/ribeirão, em $22,0 \%$ de cisterna e em 7,4\% de outras fontes; sendo que em apenas $14,7 \%$ dessas se tratava a água destinada aos animais. Quanto aos responsáveis pelas propriedades amostradas, a maioria $(72,0 \%)$ era formada por trabalhadores rurais e $27,9 \%$ possuíam outras ocupações. A renda familiar variava entre 3 e 4 salários mínimos (valor de referência: $\mathrm{R} \$$ 678,00), em 45,6\% dessas propriedades.

Após a análise de regressão logística binária simples, as variáveis que mostraram diferenças significantes entre as categorias pesquisadas foram a profissão dos indivíduos responsáveis pelas propriedades, a renda das famílias, a fonte de água e o tratamento da água das propriedades. Após essa primeira análise, as variáveis estatisticamente significantes foram submetidas à regressão logística binária multivariada, avaliando-se a associação desses fatores de risco com a prevalência de cisticercose bovina, conforme apresentados no Quadro 2.

Nesta pesquisa, os casos positivos de animais com cisticercose bovina ocorreram significativamente em maior proporção nas propriedades que não tratavam sua água (RC 9,40 IC 1,97-9,59). Essas propriedades tinham 9,4 vezes mais chances de ter cisticercose bovina quando comparadas com as propriedades que faziam uso de algum tratamento para água. Na maior parte das propriedades amostradas a qualidade da água era desconhecida; estudos mostram que a contaminação da água se relaciona entre os principais fatores de risco associados à transmissão da cisticercose bovina, uma vez que os ovos podem ser transportados pela água e ingeridos pelo animal em diferentes localidades (Boone et al. 2007, Pondja et al. 2010, Calvo-Artavia et al. 2013a).

Nas propriedades em que os responsáveis eram trabalhadores rurais (RC 5,81 IC 1,87-8,66) havia 5,8 vezes mais chances de ocorrer a doença quando comparadas com as propriedades que tinham como responsáveis indivíduos com outras profissões. No caso, o tipo de profissão se reflete nas práticas de manejo, que são identificadas como um relevante fator de risco para transmissão da cisticerco-

Quadro 1. Relação entre o número de bovinos amostrados, suspeitos (ELISA) e positivos (Imunoblot) para cisticercose bovina e os municípios pesquisados na região do Triângulo Mineiro

\begin{tabular}{lcccc}
\hline Município & Amostrados (\%) & Suspeitos (\%) & & Positivos (\%) \\
\cline { 5 - 5 } & & ELISA & & Imunoblot \\
\hline Iraí de Minas & $433(43,2)$ & $74(17,0)$ & & $21(4,8)$ \\
Romaria & $191(19,1)$ & $26(13,6)$ & & $11(5,7)$ \\
Grupiara & $172(17,2)$ & $24(14,0)$ & & $7(4,0)$ \\
Douradoquara & $206(20,5)$ & $27(13,1)$ & & $8(3,9)$ \\
TOTAL & $1002(100,0)$ & $151(15,0)$ & & $47(4,7)$
\end{tabular}

Quadro 2. Análise de regressão logística multivariada dos fatores de risco associados à transmissão da cisticercose bovina

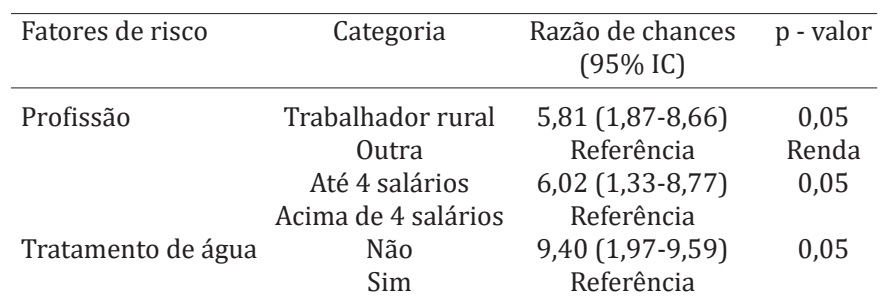

*Valor de referência para o cálculo da Razão de Chances (RC). Para o cálculo do RC foi fixado o fator de menor risco, atribuindo para tal risco 1,00 e posteriormente foi calculado a RC das variáveis tomando como referência a variável de menor risco. 
se em nível de rebanho, ou seja, o esclarecimento humano sobre a transmissão da doença pode auxiliar a prevenir a sua propagação nas criações animais (Calvo-Artavia et al. 2013c).

Os dados socioeconômicos também se mostraram relevantes, pois nas propriedades amostradas em que os indivíduos possuíam renda familiar abaixo de 4 salários mínimos (RC 6,02 IC 1,33-8,77) houve 6,02 vezes mais chances de ocorrer cisticercose nos animais quando comparado com as propriedades em que os indivíduos possuíam renda acima desse valor. Esse resultado demonstra que as baixas condições socioeconômicas podem gerar impactos negativos tanto na saúde individual quanto da comunidade na qual o indivíduo está inserido, possibilitando o aumento na transmissão de doenças (Wu et al. 2012). De acordo com Bavia et al. (2012), a forma de produção, as condições sanitárias inadequadas e o baixo nível socioeconômico contribuem maciçamente para a persistente disseminação da cisticercose.

As características da criação animal e das condições socioeconômicas nas propriedades amostradas indicaram a presença de fatores que favoreceram a transmissão da cisticercose bovina, mostrando que a prevalência encontrada na área em estudo também pode estar relacionada com esses fatores, como mostram outras pesquisas, que também identificaram a qualidade da água, o sistema de criação extensivo e semiextensivo, as baixas condições socioeconômicas da população e o tamanho da propriedade rural, entre os principais fatores, que podem contribuir para a transmissão dessa parasitose em criações animais (Asaava et al. 2009, Acevedo-Nieto et al. 2012, Calvo-Artavia et al. 2013b, Calvo-Artavia et al. 2013c, Santos et al. 2013).

A maior prevalência da doença foi apresentada no município de Romaria e as propriedades positivas desse município possuíam características que favorecem a transmissão da doença: rebanhos criados extensivamente, predomínio de criação leiteira, média de idade dos animais amostrados de 60 meses e nenhuma propriedade fazia uso de vermífugos para endoparasitas em seus animais. De acordo com Calva-Artavia et al. (2013b), rebanhos leiteiros têm maior proporção de animais positivos quando comparado com outros tipos de produção, podendo os animais serem expostos a ovos de T. saginata na pastagem várias vezes durante a sua permanência na propriedade. A soro prevalência da cisticercose bovina também tem sido positivamente correlacionada com o aumento da idade, elevando-se o risco de infecção com o avanço na idade dos animais, dada a sua maior exposição aos fatores de risco como pastagens ou águas contaminadas (Boone et al. 2007).

0 abate clandestino é um outro fator preponderante na disseminação de doenças, sendo uma prática nociva e insegura à saúde humana (Mellau et al. 2010). Embora, esse fator não tenha apresentado significância estatística nos critérios analíticos adotados neste estudo, observou-se que apenas $29,4 \%$ dos bovinos criados nas propriedades eram destinados ao abate inspecionado. Um outro estudo, realizado em um município da zona da mata mineira, também verificou que as propriedades realizavam abate clandestino, mostrando ser uma característica comum nas propriedades rurais (Santos et al. 2013).

\section{CONCLUSÕES}

0 presente estudo permitiu detectar a existência da cisticercose bovina nas propriedades rurais amostradas nos quatro municípios do Triângulo Mineiro, revelando uma prevalência da doença de 4,7\%.

0 perfil da transmissão da doença está centrado no sistema de criação dessas propriedades no qual os animais tem livre acesso as águas de qualidade desconhecida.

Outros estudos a campo, não apenas na região do Triângulo Mineiro como também em outras regiões do Brasil, com as peculiaridades da criação animal em cada região, devem ser realizadospara que se possa identificar mais fatores de risco relacionados com a transmissão da cisticercose a fim de se traçar estratégias de controle, garantindo dessa forma, a saúde das famílias nas áreas rurais e a saúde dos animais.

Agradecimentos. - Ao Instituto Mineiro de Agropecuária (IMA), pela cooperação logística, aos produtores rurais pela colaboração. À Coordenação de Aperfeiçoamento de Pessoal de Nível Superior (CAPES), pela concessão de bolsa de mestrado, ao Conselho Nacional de Desenvolvimento Científico e Tecnológico (CNPq) e a Fundação de Amparo à Pesquisa do Estado de Minas Gerais (FAPEMIG).

\section{REFERÊNCIAS}

Almeida L.P., Moreira M.D., Reis D.O. \& Santos W.L.M. 2002. Cisticercose bovina: um estudo comparativo entre animais abatidos em frigoríficos com serviço de inspeção federal e com inspeção municipal. Revta Hig. Alim. 16(99):51-55.

Almeida L.P., Reis D.O., Moreira M.D. \& Palmeira S.B.S. 2006. Cisticercose em bovinos procedentes de Minas Gerais e abatidos em frigoríficos de Uberlândia-MG, no período de 1997 a 2001. Revta Hig. Alim. 2(139):40-43.

Almeida L.P. 2012. O Projeto de Pesquisa - Passo a Passo. Assis Editora, Uberlândia. 232p.

Acevedo-Nieto E.C., Vieira F.C., Pinto P.S.A., Silva L.F., Santos T.O. \& Peixoto R.P.M.G. 2012. Análise de fatores de risco para a infecção de cisticercose bovina: estudo de caso controle a partir de animais abatidos. Semina, Ciênc. Agrárias 33(6):2359-2366.

Asaava L.L., Kitala P.M., Gathura P.B., Nanyingi M.O., Muchemi G. \& Schelling E. 2009. A survey of bovine cysticercosis human taeniosis in Northern Turkana District, Kenya. Prev. Vet. Med. 89:197-204.

Bavia M.E., Carneiro D.D.M.T., Cardim L.L., Silva M.M.N. \& Martins M.S. 2012. Estatística espacial de varredura na detecção de áreas de risco para a cisticercose bovina no estado da Bahia. Arq. Bras. Med. Vet. Zootec. 64(5):1200-1208.

Boone I., Thys E., Marcotty T., Borchgrave J.D., Ducheyne E. \& Dorny P. 2007. Distribution and risk factors of bovine cysticercosis in Belgian dairy and mixed herds. Prev. Vet. Med. 82:1-11.

Calvo-Artavia F.F., Nielsen L.R., Dahl J., Clausen D.M. \& Alban L. 2013a. Occurrence and factors associated with bovine cysticercosis recorded in cattle at meat inspection in Denmark in 2004-2011. Prev. Vet. Med. 110:177-182.

Calvo-Artavia F.F., Nielsen L.R. \& Alban L. 2013b. Epidemiologic and economic evaluation of risk-based meat inspection for bovine cysticercosis in Danish cattle. Prev. Vet. Med. 108:253-261.

Calvo-Artavia F.F., Nielsen L.R., Dahl J., Clausen D.M., Graumann A.M. \& Alban L. 2013c. A case-control study of risk factors for bovine cysticercosis in Danish cattle herds. Zoonoses Public Health 60:311-318.

CDC 2013. Center for Disease Control and Prevention. Cysticercosis. Disponível em: <http://www.dpd.cdc.gov/dpdx/HTML/Cysticercosis. htm>. Acesso em: 07 nov. 2013.

Deckers N. \& Dorny P. 2010. Imunodiagnosis of Taenia solium taeniosis/ cisticercosis. Trends Parasitol. 26(3):137-144. 
Eichenberger R.M., Stephan R. \& Deplazes P. 2011. Increased sensitivity for the diagnosis of Taenia saginata cysticercus infection by additional heart examination compared to the EU-approved routine meat inspection. Food Control 22:989-992.

IBGE 2014a. [Census of the Brazilian Institute of Geography and Statistics]: cidades. Instituto Brasileiro de Geografia e Estatística. Disponível em <http://cidades.ibge.gov.br/xtras/home.php> Acesso em 2 mar. 2014.

IBGE 2014b. [Census of the Brazilian Institute of Geography and Statistics]: população. Instituto Brasileiro de Geografia e Estatística. Disponível em: < http://www.ibge.gov.br/home/estatistica/populacao/censo2010/default.shtm> Acesso em 3 abr. 2014.

Ogunremi O. \& Benjamin J. 2010. Development and field evaluation of a new serological test for Taenia saginata cysticercosis. Vet. Parasitol. 169:93-101.

Oliveira J.T.F., Medeiros E.F., Porto W.J.N., Pinheiro Junior J.W., Alves L.C., Silva P.G. \& Soares K.D.A. 2013. Prevalência da cisticercose bovina em estabelecimento sob inspeção federal no município de Promissão, SP. Revta Hig. Alim. 27(220/221):94-97.

Pondja A., Neves L., Mlangwa J., Afonso S., Fafetine J., Willingham A.L., Thamsborg S.M. \& Johansen M.V. 2010. Prevalence and risk factors of porcine cysticercosis in Angónia District, Mozambique. Plos Negl. Trop. Dis. 4:1-5.

Pinto P.S.A., Vaz A.J., Germano P.M.L. \& Nakamura P.M. 2000. Performance of the ELISA test for swine cysticercosis using antigens of Taenia solium and Taenia crassiceps cysticerci. Vet. Paratol. 88:127-130.

Pinto P.S.A., Vaz A.J., Nakamura P.M. \& Germano P.M. L. 2001. Immunoblot analysis using antigen from Taenia crassiceps cysticerci in the diagnosis of swine cysticercosis. Vet. Parasitol. 56:36-42.
Mellau L.S.B., Nonga H.E. \& Karimuribo E.D. 2010. A slaughterhouse survey of lung lesions in slaughtered stock sat Arusha, Tanzania. Prev. Vet. Med. 97:77-82.

Monteiro L.L., Pinto P.S.A. \& Dias F.S. 2006. Evaluation of the ELISA test for the antibody detection in catle naturally and experimentally infected with Cysticercus bovis. Vet. Parasitol. 141:260-263.

Rey L. 2008. Tênias e teníases, p.516-539. In: Rey L. (Ed.), Parasitologia: parasitos e doenças parasitárias do homem nos trópicos ocidentais. $4^{\text {a }}$ ed. Guanabara Koogan, Rio de Janeiro.

Santos J.M.G. \& Barros M.C.R.B. 2009. Cysticercus bovis e Cysticercus cellulosae: endoparasitas de importância no comercio de carne. Revta Agron. Meio Amb. 2(1):21-39.

Santos T.O., Pinto P.S.A., Iasbik A.F., Silva L.F., Acevedo-Nieto E.C. \& Guimarães-Peixoto R.P.M. 2013. Epidemiological survey of the taeniasis/cysticercosis complex in cattle farms in Viçosa County, Minas Gerais, Brazil. Pesq. Vet. Bras. 33(4):449-452.

Scandrett B., Parker S., Forbes L., Gajadhar A., Dekumyoy P., Waikagul J. \& Haines D. 2009. Distribution of Taenia saginata cysticerci in tissues of experimentally infected cattle. Vet. Parasitol.164:223-231.

Silva A.V.M. 2005. Teníase e cisticercose, p.227-237. In: Ibid. (Ed.), Parasitologia Humana. 11 $1^{\mathrm{a}}$ ed. Atheneu, São Paulo.

WHO 2008. EPI INFO Database and statistics software for public health professionals. Centers for Disease Control and Prevention (CDC). Versão 3.5.1.

Wu W., Qian X., Huang Y. \& Hong Q. 2012. A review of the control of clonorchiasis sinensis and Taenia solium taeniasis/cysticercosis in China. Parasitol. Res. 111:1879-1884. 